\title{
A Large Outbreak of Legionnaires' Disease Due to an Inadequate Circulating and Filtration System for Bath Water-Epidemiologic Manifestations-
}

\author{
Hiroyuki NAKAMURA, Hisanaga YAGYU, Koji KISHI, Fumihiro TsUCHIDA, \\ Shuji OH-ISHI, Keizo YAMAGUCHI* and Takeshi MATSUOKA
}

\begin{abstract}
Objective To study the epidemiologic manifestations of a large outbreak of Legionnaires' disease due to an inadequate circulating and filtration system for bath water.

Patients In June 2000 at Ishioka City, Ibaraki Prefecture, a large outbreak of Legionnaire's disease occurred, as a result of an inadequate circulating and filtration system for communal bath water. This outbreak was the worst ever experienced in Japan, involving a total of 34 patients ( 20 confirmed, 14 probable), 3 of whom died.

Measurements and Results Legionella pneumophila serogroup 1 was isolated from sputum culture in two patients. Bacteriological culture of the public bath water subsequently yielded large numbers of Legionella species. Cleavage of genomic DNA showed that restriction fragment patterns coming from clinical and environmental isolates of $L$. pneumophila serogroup 1 were closely related, focusing the inquiry to a public bathhouse where a circulating filtration system was suspected as the source of infection.

Conclusions It was later concluded that the circulating filtration system adopted for bath water was marred by a serious design flaw that subsequently caused the mass outbreak. Specifically, a line of the bath water was being returned to the bath without undergoing heat exchange or sterilization by chlorine; and the Legionella species that had proliferated in the filter and the bright stone filtration unit were allowed to return to the bath, eventually culminating in a mass infection.

(Internal Medicine 42: 806-811, 2003)
\end{abstract}

Key words: Legionnaires' disease, large outbreak, public bathhouse, circulating bath water system, molecular epidemiology

\section{Introduction}

The Legionella species (Lsp), a Gram-negative rod, is widely distributed in water and soil; and is responsible for a respiratory infection when contaminated aerosols are inhaled (1). Legionnaires' disease is a febrile illness with pneumonia caused by the Lsp. Large numbers of epidemic and sporadic cases have been reported $(1,2)$. In this instance the large outbreak of Legionnaires' disease was due to an inadequate circulation and filtration system for bath water in which 34 individuals who had become infected, had visited the same public bathhouse. Epidemiological details of the incidence are reported here so that similar events may be averted in the future.

\section{Materials and Methods}

\section{Microbiological investigations}

Growth conditions and isolation of Legionella pneumophila (L. pneumophila)

Water samples and environmental swabs were collected and placed into sterile containers from 23 sites, including hot water, sinks and sauna rooms, water fountains, water storage tanks and elements of the bathing facility circulation systems (tubs, drains, storage tanks, and filters). These samples were concentrated by filtration or centrifugation and cultured on buffered charcoal-yeast extract agar containing $0.1 \%$ alpha-ketoglutarase (BCYE $\alpha$ ) (Eiken Chemical; Tokyo, Japan) (3).

From the Fifth Department of Internal Medicine, Tokyo Medical University, Ibaraki and *Department of Microbiology, Toho University School of Medicine, Tokyo

Received for publication January 24, 2003; Accepted for publication April 28, 2003

Reprint requests should be addressed to Dr. Hiroyuki Nakamura, the Fifth Department of Internal Tokyo Medical University, 3-20-1 Chuo Ami-cho, Inashiki, Ibaraki 300-0395 
Clinical specimens such as sputum, bronchoalveolar lavage fluid, serum and urine from patients suspected of legionellosis were sent to Toho University School of Medicine. Respiratory specimens containing mucus were liquefied with semi-alkaline protease (Kyokutoh Pharmaceutical; Tokyo, Japan) and incubated at $37^{\circ} \mathrm{C}$ for 10 minutes. Specimens were then centrifuged; half were heat treated, the other half inoculated directly onto BCYE $\alpha$ and Wadowsky-Yee-Okuda (WYO $\alpha$ ) (Eiken Chemical) agar plates with antibiotics. The plates were incubated at $35^{\circ} \mathrm{C}$ with 70 to $80 \%$ relative humidity and were examined for 3 to 10 days. Visible colonies subcultured onto a BCYE $\alpha$ agar plate and a WYO $\alpha$ agar plate were considered as possible Lsp and were tested by direct fluorescent-antibody assay (DFA) (Denka Seiken; Tokyo, Japan) for Legionella pneumophila serogroup 1 (Lp1) to Lp6, Legionella bozemanii (L. bozemanii), Legionella dumoffii ( $L$. dumoffii), Legionella gormanii (L. gormani), and Legionella micdadei (L. micdadei). When DFA with the isolates were negative, identification was made by the microplate DNA-DNA hybridization method (Kyokutoh Pharmaceutical) (4).

Search for Pseudomonas aeruginosa, non-tuberculous mycobacterium and amoeba from water samples and environmental swabs were not implemented.

\section{Detection of L. pneumophila antigens in urine samples}

Legionella antigens in urine samples were examined by BinaxNOW immunochromatographic assay (ICT) (Binax; Portland, OR, USA), Binax Legionella Urinary Enzyme Immunoassay (EIA) (Binax) and Biotest Legionella Urine Antigen EIA (Biotest; Dreieich, Germany). Both the BinaxNOW ICT assay and Binax EIA are marketed by the manufacturers as kits for detection of Lp1 urinary antigen. Conversely, Biotest Legionella Urine Antigen EIA is marketed as recognizing all serogroups of L. pneumophila (5).

\section{Determination of serum antibody titer}

Serum antibody titers were examined by microplate agglutination test (MPAT) (Denka Seiken; Tokyo, Japan), as described previously $(6,7)$. Briefly, serum samples were decomplemented by heat treatment $\left(56^{\circ} \mathrm{C}, 30\right.$ minutes $)$ and then serially diluted in PBS from 1 in 8 to 1 in 512 in the wells of a U-bottom microtitration plate (25- $\mu$ l volumes). Heat-killed bacteria, including L. pneumophila (serogroups 1-6), L. bozemanii, L. dumoffii, L. gormani and L. micdadei, were prepared by incubating bacterial suspensions for 1 hour at $100^{\circ} \mathrm{C}$, and the concentration adjusted to an OD650 of 0.3 . Heat-killed bacterial suspensions $(25 \mu \mathrm{l})$ were inoculated into each well of diluted serum samples. The plate was incubated at room temperature for 20 hours, after which agglutination in the wells was determined.

\section{Method of polymerase chain reaction $(P C R)$}

The procedure can be performed in 6 to 8 hours with a commercially available DNA extraction kit (Qiagen; Valencia, CA) and by PCR with gel detection (8). PCR is performed with primers previously determined to amplify a 386-bp product within the $16 \mathrm{~S}$ rRNA gene of Lsp in respiratory samples. Several medically relevant Lsp, including $L$. pneumophila, L. micdadei, L. bozemanii, L. dumoffii, Legionella longbeachae, and Legionella feeleii can be detected by this method.

\section{Chromosomal analysis by pulsed-field gel electrophoresis}

Genomic DNA for pulsed-field gel electrophoresis (PFGE) analysis was prepared by using the GenePath plug Kit 5 (Bio-Rad Laboratories; Hercules, CA, USA) by following the manufacturers' protocol $(9,10)$. Two hundred microliters of overnight cultures of the L. pneumophila isolates grown in BCYE $\alpha$ broth were centrifuged at 8,500 $\mathrm{g}$ for 5 minutes. The supernatant was discarded, and the sediments resuspended in a $150 \mu \mathrm{l}$ cell suspension buffer. Immediately following that, $150 \mu \mathrm{l}$ of liquid $1.2 \%$ embedding agarose and $6 \mu \mathrm{l}$ lysozyme were mixed with the suspension and cooled to $50^{\circ} \mathrm{C}$. The mixture was poured into plug molds on ice. The plugs were then incubated in a $500 \mu$ lysis buffer containing $20 \mu \mathrm{l}$ lysozyme for 1 hour at $37^{\circ} \mathrm{C}$. After serial treatment with proteinase $\mathrm{K}$ and washing buffer, one-third of each plug was digested with the restriction enzyme SfiI (5 U) in 300 $\mu \mathrm{l}$ SfiI buffer at $50^{\circ} \mathrm{C}$ for 16 hours. Fragments of DNA were separated on $1 \%$ SeaKem Gold agarose gel (FMC Bioproducts; Rockland, MD) in $1 \times \mathrm{TBE}$ buffer at $14^{\circ} \mathrm{C}$ for 19.7 hours with a switch ramped time from 5.3 to 49.9 seconds at a $120^{\circ}$ angle on a CHEF MAPPER apparatus (Bio-Rad Laboratories). The sizing ladder used for PFGE was the lambda ladder 170-3,635 with a range of from 0.05 to $1 \mathrm{MB}$ (Bio-Rad Laboratories). The PFGE patterns were compared using the Dendron software (Solltech Inc.; Oakdale, LA, USA). Dendrograms based on a similarity coefficient $\left(\mathrm{S}_{\mathrm{AB}}\right)$ were created with the Dendron software program by the unweighted pair group matching average (UPGMA).

\section{Criteria for confirmed diagnosis}

Prima fascia evidence of Legionnaires' disease was defined as physician-diagnosed pneumonia with laboratory evidence of legionella infection in individuals that used the same bathing facility. Laboratory evidence of Legionnaires' disease included isolation of legionellae from respiratory secretions, detection of $L$. pneumophila antigens in urine, detection of Lsp DNA by PCR, or a four-fold rise in Lsp antibody titer to 1 in 128 or more in paired acute-phase serum samples.

\section{Results}

\section{Demographic data, risk factors and identification method for definitive diagnosis}

Demographic data, risk factors and identification method for definitive diagnosis of patients with pneumonia caused by Lsp are shown in Table 1. Thirteen males and 7 females developed Legionnaires' disease. The mean age was 62.2 years (range, 27 to 85 years). The incubation period was 5.3 
Table 1. Demographic Data Risk Factors and Identification Methods for Definitive Diagnosis of Patients with Pneumonia Caused by Legionella Species

Gender Male; 13, Female; 7

Mean age 62.2 years (range, 27 to 85 years)

Incubation period 5.3 days (range, 2 to 9 days)

Incidence rate $0.12 \%$

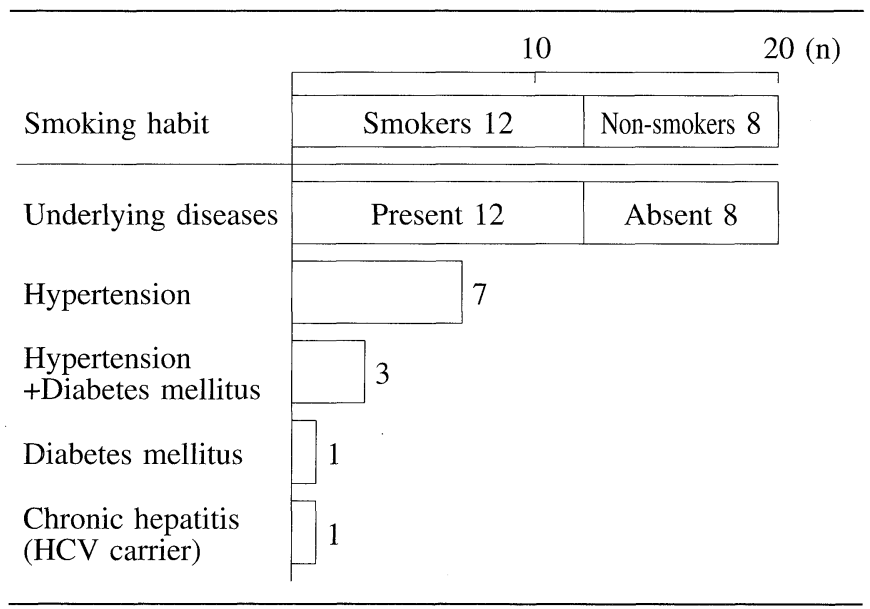

Confirmed 20

Diagnostic methods for confirmed cases; Antibody titer 14/20, Culture $2 / 20$, Urinary antigens $4 / 20$, Polymerase chain reaction 7/20 (Sputum; 3, Urine; 1, Serum; 1, Urine+serum; 1, Pleural effusion; 1)

Frequency of different Legionella pneumophila serogroup and Legionella species

Lp1; 12, Lp6; 2, Lp1+Lp6; 4, Legionella species; 2

Lp: Legionella pneumophila serogroup.

days (range, 2 to 9 days). During the same period, 16,267 people used the same bathing facility. The incidence was therefore computed to be $0.12 \%$. Unfortunately age and sex distributions of 16,267 cases taking a bath was uncertain. Among those patients that developed Legionnaires' disease, 12 were smokers. Furthermore 12 patients reported underlying diseases, none of which were considered serious. Twenty patients had confirmed Legionella pneumonia; as diagnosed by serum antibody titer (14 cases), culture ( 2 cases), detection of urinary antigen (4 cases) or PCR (7 cases) or combination of the above. In the confirmed cases, the etiologic species were considered to be Lp1 in 12 cases, Lp6 in 2 cases and Lp1 plus Lp6 in 4 cases, because the antibody titer to both were elevated. Among the confirmed cases, one patient died due to a respiratory failure.

\section{Design of the public bathhouse and detection of Lsp}

The design of the public bathhouse and detection of Lsp are shown in Fig. 1. The bathhouse was divided into 2 areas: the FU-1 area that was composed of an indoor bathtub, a waterfall, mist-sauna room, and outdoor bath; and the FU-2 area, consisted of an indoor bath, soaking tub, a dry sauna, and outdoor jacuzzi. L. pneumophila (Lp1, Lp5, and Lp6)
[8.42 $\times 10^{3}$ colony forming units $\left.(\mathrm{CFU}) / 100 \mathrm{ml}\right]$ were isolated from the indoor bathtub in the FU-1 area. The diluents of the samples collected from the surface structures of the mist sauna yielded Lp1, Lp5, and Lp6. The indoor bathtub in the FU-2 area produced Lp1 and Lp5.

\section{Design of the circulation-filtration system and detec- tion of Lsp}

The design of the circulation-filtration system and detection of the Lsp are shown in Fig. 2. The designs of the FU-1 and FU-2 areas for the circulation and filtration of bath water were identical. Specifically, the circulating bath water was composed of one line that was returned directly to the tub after simply flowing through the filter and through the bright stone filtration unit, and a second line that was returned to the tub after sterilization by heat and chlorine.

The parts of the filter in the FU-1 area yielded Lp1 and Lp6; and the filter component of the bright stone filtration unit, Lp6. The water that had been filtered in the FU-2 area produced $1.14 \times 10^{3} \mathrm{CFU} / 100 \mathrm{ml}$ of $L$. pneumophila (Lp1, Lp3, Lp5, and Lp6).

\section{UPGMA cluster analysis}

Dendrogram showing UPGMA cluster analysis of pairwise comparisons of similarity between the PFGE patterns of L. pneumophila isolates from two patients and environment are shown in Fig. 3. It was confirmed that there was a high degree of homology among the DNA breakage patterns of clinical isolates 1 and 2 and those isolated from environments, $3,4,5,6,7,8$, and 10 , confirming the suspicion that they shared a single infecting source.

\section{Discussion}

Mass outbreaks of legionellosis originating in bathing facilities have been reported in the United States $(11,12)$ and France (13). In Japan, incidences of Legionnaires' disease with the causative agents probably originating in spas have been described $(14,15)$ and there is a reported outbreak involving 3 patients in Iwate Prefecture in 1999 (16). Thus we concluded that the present outbreak was the largest mass epidemic of legionellosis, involving a bathhouse as a source of infection, in which heated water was continuously circulated. The homology between the 2 strains isolated from clinical patients was less than $70 \%$ when based on the results of PFGE and computed by using Jaccard's coefficient and UPGMA. However, these 2 strains exhibited $70 \%$ or more homology with the strains with Lp1 and Lp3 that originated from the environment. It is accepted that a homology of $70 \%$ or more indicates that the organisms share a single origin. In the present investigation, the molecular homology of Lp1 that had been isolated from clinical patients and bath water proved that they were indeed from a single source. Among the other serogroups isolated from the environment, the two strains belonging to Lp5 showed 100\% homology, but those belonging to Lp6 were found to have originated from three 
A Large Outbreak of Legionnaires’ Disease

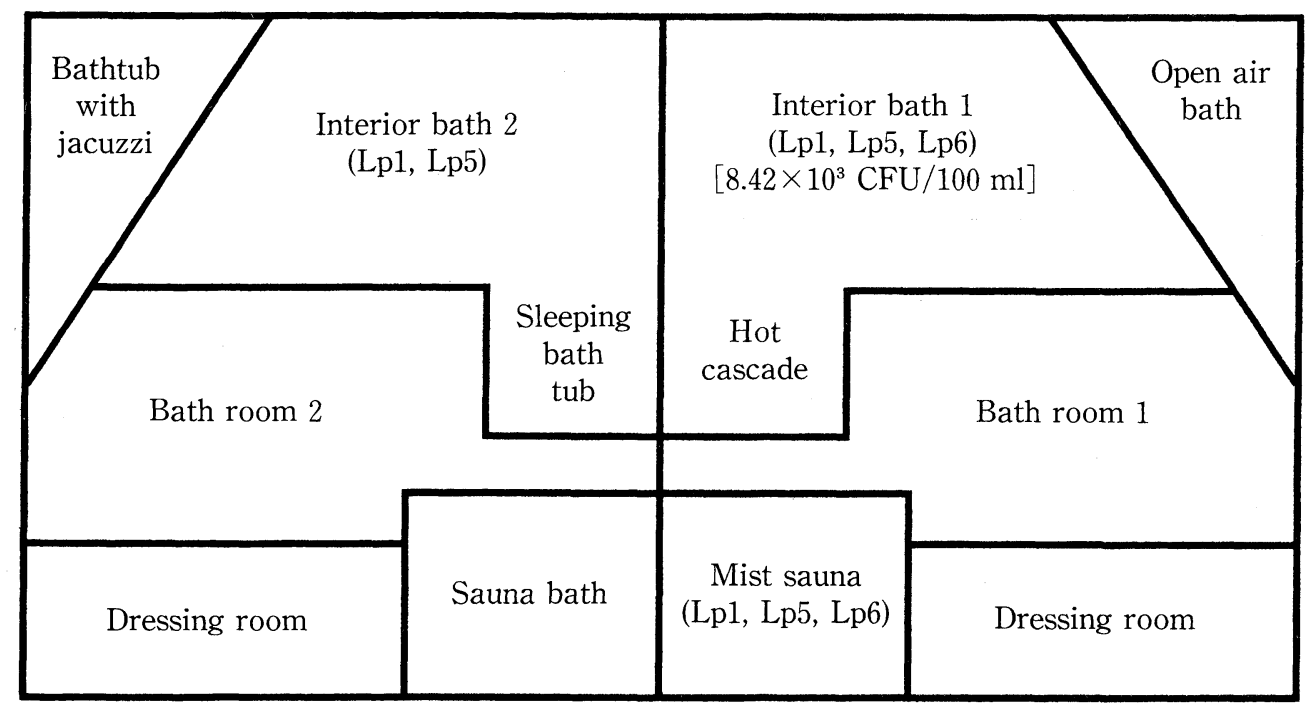

(FU-2 area)

(FU-1 area)

Figure 1. Diagram of the bathing facility and isolation of Legionella pneumophila serogroup. CFU: colony forming units.
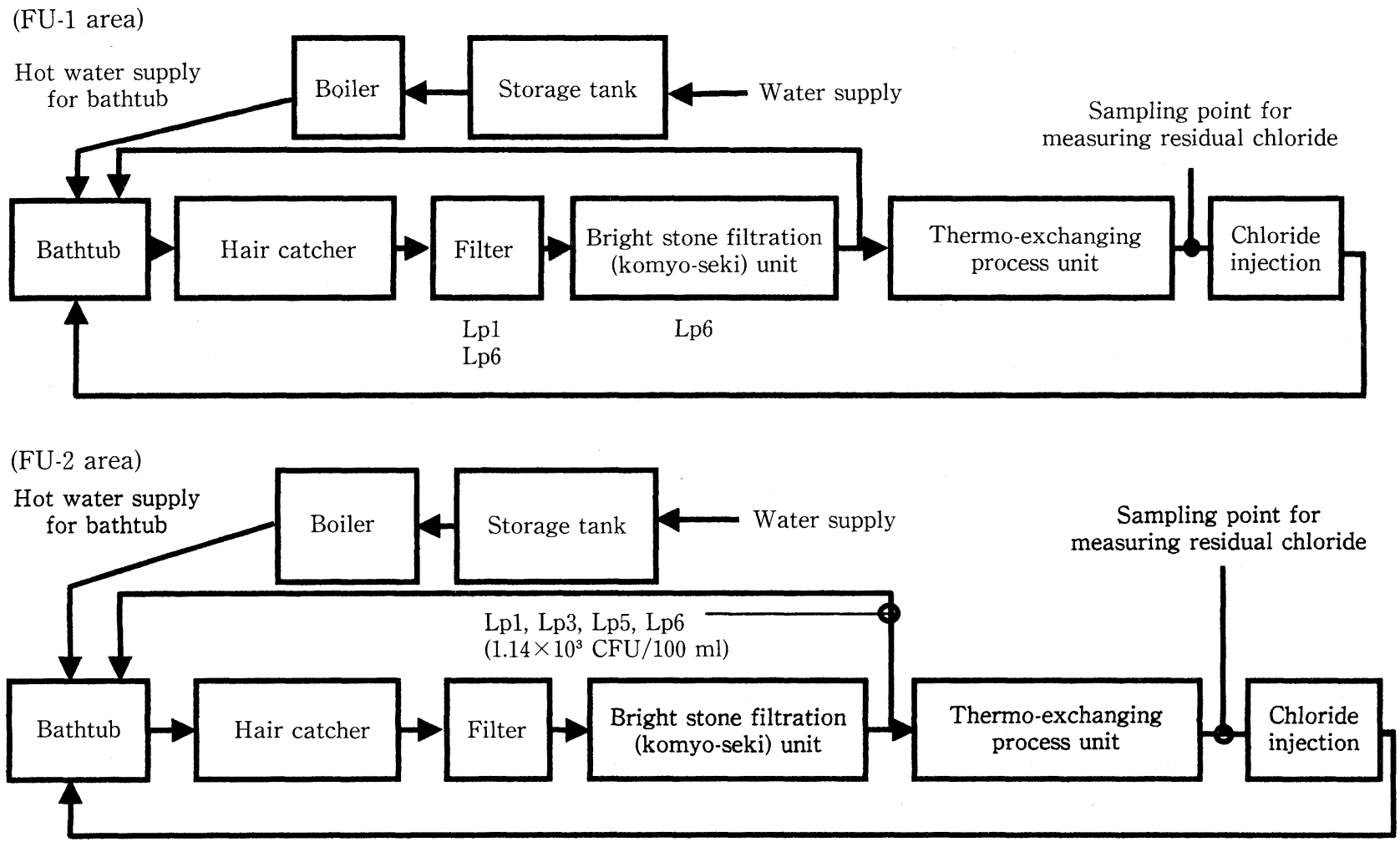

Figure 2. Flowchart of circulation and filtration line of bath water (interior bath) and isolation of Legionella species and Legionella pneumophila serogroup. See Table 1 and Fig. 1 for abbreviations not used in the text. 


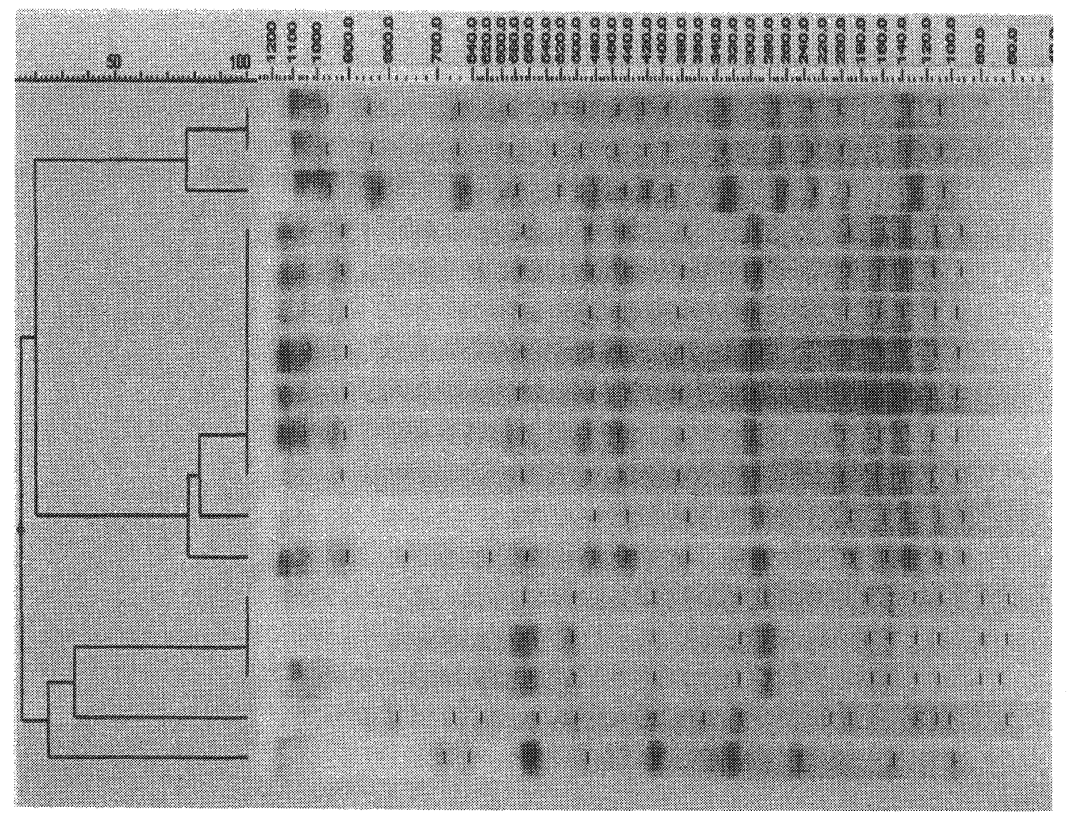

$\begin{array}{cr}\begin{array}{c}\text { Sample } \\ \text { numbers }\end{array} & \text { Serog } \\ 18 & 6 \\ 19 & 6 \\ 21 & 6 \\ 3 & 1 \\ 4 & 1 \\ 5 & 1 \\ 6 & 1 \\ 7 & 1 \\ 8 & 1 \\ 10 & 3 \\ 1 & 1 \\ 2 & 1 \\ 13 & 5 \\ 14 & 5 \\ 16 & 6 \\ 20 & 6 \\ \text { ATCC33152 } & 1\end{array}$

\begin{abstract}
Figure 3. Dendrogram showing UPGMA cluster analysis of pair-wise comparisons of similarity between the PFGE patterns of Legionella pneumophila isolates from two patients and environment. PFGE profile using SfiI to digest genomic DNAs from Legionella species isolated from two patients and a public bathhouse. Sample numbers: 1 and 2 were isolated from two patients. Others were isolated from the public bathhouse. ATCC33152: strains of Legionella pneumophila serogroup 1 (Philadelphia 1). Serogroup: The number was indicated serogroup of Legionella pneumophila.
\end{abstract}

different sources. It was also discovered that Lp1 and Lp3, Lp5 and Lp6 were interrelated. Thus it was concluded that there are five strains originating from different sources among those isolated from the environments. Although bacterial serotyping continues to be a useful tool for epidemiologic studies, there is a need for a method of strain typing that can be used to type a broader array of bacterial species. At present, PFGE comes closest to satisfying that need (9, 17).

The water line that returned to the bath directly, without heat exchange or chlorine sterilization also supplied a waterfall at a height of $139 \mathrm{~cm}$. Furthermore, the bath was equipped with a device resembling a jet or air vibrator to generate an aerosol. It was presumed that a large amount of bacteria was aerosolized and inhaled by the bathers, resulting in the mass infection of the individuals. Additionally it was further observed that there were many other management problems evident such as inadequate sterilization and replacement of the water used for bathing.

Furthermore, in association with changes in living conditions, it is expected that circulating type baths and 24-hour hot water baths will become increasingly more popular. It is mandatory that safe circulating filtration systems be adopted for bath water and that such systems be inspected and conform to safety and health regulations. Furthermore, not only clinicians, but also designers and managers of bathing facilities and equipment, those involved with related devices, and also the general public should be made aware of the fact that we are constantly exposed to the risk of contracting an infection by Lsp. Legislative measures should include health education for citizens, as well as implementation of methods for thorough inspection and testing of related facilities so that incidents such as that reported here may never again occur in the future.

\section{References}

1) Fraser DW, Tsai TR, Orenstein W, et al. Legionnaires' disease: Description of an epidemic of pneumonia. N Engl J Med 297: 11891197, 1977.

2) England AC, Fraser DW, Plikaytis BD, Tsai TF, Storch G, Broome CV. Sporadic legionellosis in the United States: the first thousand cases. Ann Intern Med 94: 164-170, 1981.

3) Edelstein PH. Improved semiselective medium for isolation of Legionella pneumophila from contaminated clinical and environmental specimens. J Clin Microbiol 14: 298-303, 1981.

4) Ezaki $T$, Hashimoto $Y$, Yamamoto $H$, et al. Evaluation of the microplate hybridization method for rapid identification of Legionella species. Eur J Clin Microbiol Infect Dis 9: 213-217 1990.

5) Helbig JH, Uldum SA, Luck PC, Harrison TG. Detection of Legionella pneumophila antigen in urine samples by the BinaxNOW immunochromatographic assay and comparison with both Binax Legionella Urinary Enzyme Immunoassay (EIA) and Biotest Legionella Urin Antigen EIA. J Med Microbiol 50: 509-516, 2001.

6) Yabuuchi E, Saito A, Niki Y, et al. Determination of cutoff value of 


\section{A Large Outbreak of Legionnaires' Disease}

serum anti-Legionella antibody titre, microplate agglutination test (MPAT)-. Kansenshogaku Zasshi 71: 116-124, 1997 (in Japanese, Abstract in English).

7) Tateda K, Murakami H, Ishii Y, Furuya N, Matsumoto T, Yamaguchi $\mathrm{K}$. Evaluation of clinical usefulness of the microplate agglutination test for serological diagnosis of legionella pneumonia. J Med Microbiol 47: 325-328, 1998.

8) Cloud JL, Carroll KC, Pixton P, Erali M, Hillyard R. Detection of Legionella species in respiratory specimens using PCR with sequencing confirmation. J Clin Microbiol 38: 1709-1712, 2000.

9) Tenover FC, Arbeit RD, Goering RV, et al. Interpreting chromosomal DNA restriction patterns produced by pulsed-field gel electrophoresis: criteria for bacterial strain typing. J Clin Microbiol 33: 2233-2239, 1995.

10) Sneath PHA, Sokal RR. Numerical taxonomy in: The Principles and Practice of Numerical Classification. WH Freeman \& Co, San Francisco, 1973: 230-234.

11) Jernigan DB, Hofmann J, Cetron MS, et al. Outbreak of Legionnaires' disease among cruise-ship passengers exposed to a contaminated whirlpool spa. Lancet 347: 494-499, 1996.

12) Centers for Disease Control. Legionnaires disease associated with a whirlpool spa display-Virginia, September-October, 1996. MMWR 46:
83-86, 1997.

13) Bornstein N, Marmet D, Surgot M, et al. Exposure to Legionellaceae at a hot spring spa: a prospective clinical and serological study. Epidemiol Infect 102: 31-36, 1989.

14) Shiota R, Takeshita K, Yamamoto K, Imada K, Yabuuchi E, Wang L. Legionella pneumophila serogroup 3 isolated from a patient of pneumonia developed after near drowning in bath tub of a hot spring spa. Kansenshogaku Zasshi (J Jpn Assoc Infect Dis) 69: 1356-1364, 1995 (in Japanese, Abstract in English).

15) Mashiba K, Hamamoto T, Torikai K. A case of Legionnaires' disease due to aspiration of hot spring water and isolation of Legionella pneumophila from hot spring water. Kansenshogaku Zasshi (J Jpn Assoc Infect Dis) 67: 163-166, 1993 (in Japanese, Abstract in English).

16) Nakadate $T$, Yamauchi $K$, Inoue $H$. An outbreak of Legionnaire's disease associated with a Japanese spa. Nihon Kokyuki Gakkai Zasshi (Jpn J Thorac Dis) 37: 601-607, 1999 (in Japanese, Abstract in English).

17) Arbeit RD. Laboratory procedures for the epidemiologic analysis of microorganisms. in: Manual of Clinical Microbiology. 7th ed. Murray PR, Baron EJ, Pfaller MA, et al, Eds. ASM PRESS, Washington, 1999: 116-137. 